\title{
Vector-Sensor Array Processing for Polarization Parameters and DOA Estimation
}

\author{
Caroline Paulus and Jérôme I. Mars \\ GIPSA-Lab, Département Images Signal, 961 rue de la Houille Blanche, BP 46, 38402 Saint Martin d'Hères Cedex, France
}

Correspondence should be addressed to Jérôme I. Mars, jerome.mars@gipsa-lab.grenoble-inp.fr

Received 13 July 2009; Revised 1 February 2010; Accepted 2 May 2010

Academic Editor: Kostas Berberidis

Copyright ( $) 2010$ C. Paulus and J. I. Mars. This is an open access article distributed under the Creative Commons Attribution License, which permits unrestricted use, distribution, and reproduction in any medium, provided the original work is properly cited.

\begin{abstract}
This paper presents a method allowing a complete characterization of wave signals received on a vector-sensor array. The proposed technique is based on wavefields separation processing and on estimation of fundamental waves attributes as the state of polarization state and the direction of arrival. Estimation of these attributes is an important step in data processing for a wide range of applications where vector sensor antennas technology is involved such as seismic processing, electromagnetic fields studies, and telecommunications. Compared to the classic techniques, the proposed method is based on computation of multicomponent wideband spectral matrices which enable to take into account all information given by the vector sensor array structures and thus provide a complete characterization of a larger number of sources.
\end{abstract}

\section{Introduction}

Over the past decade, the use of vector-sensor array (VSA) technology for source localization has significantly increased allowing a better characterization of the recorded phenomena in a wide range of applications (e.g., acoustics, electromagnetism, radar, sonar, geophysics, etc.) [1-5]. For instance in seismic acquisition case, vector sensors are nowadays widely used, allowing a better characterization of the layers thanks to the state of polarization dimension added to detection process. With a vector sensor, we can have access to the particle-displacement vector that describes the particle motion in $3 \mathrm{D}$ at a given point in space. As the state of polarization is wavefield dependent, it can be used as an essential attribute to separate waves in addition to their different DOAs. To resume, multicomponent acquisitions provide more detailed information on the recorded wavefield and VSA-recorded signals allow the estimation of the directions of arrival (DOA) and the polarizations of multiple waves (or sources) impinging the array. In the case of elastic and acoustic seismic surveys, the VSA-recorded signals are a mixture of various wave types (body waves, surface waves, converted waves, multiples, noise, etc.). Combined multicomponent acquisition and multicomponent processing and analysis provide better wave characterizations and enhance the imaging resolution of geological features. In order to perform the characterization of each wave, separation of interfering wavefields is a crucial step. In the case of multicomponent sensor arrays, methods of filtering, of source localization, and of polarization estimation have already been developed for acoustics and electromagnetic sources. In the last decade, many array processing techniques for source localization and polarization estimation using vector sensors have been developed, mainly in electromagnetics. Nehorai and Paldi [1] proposed the Cramer-Rao bound and the vector cross-product DOA. Li and Compton Jr [3] developed the ESPRIT algorithm for a vector-sensor array. MUSIC-based algorithms were also proposed by Wong and Zoltowski [6-8], who also developed vector-sensors versions of ESPRIT [9-13]. These approaches represent a highly popular subspace-based parameter estimation method and use matrix techniques directly derived from scalar-sensor array processing. Such a method is based on the long-vector approach, consisting in the concatenation of all components of the vector-sensor array in a long vector [9].

The originality of our method consists in keeping multidimensional structures of data organization for processing. These structures are more adapted to the nature of 
seismic polarized signals, allowing data organization closer to its multimodal intrinsic structure. This paper presents a novel subspace separation method performing wavefields separation. This method issued from the Multicomponent Wideband Spectral Matrix Filtering (MWSMF) technique $[14,15]$ is a subspace separation algorithm derived from the classic spectral matrix filtering presented in $[16,17]$. After a separation step where each wavefield has been isolated, we propose polarization and DOA estimations for each separated wavefield that takes all frequencies and all components into account. The algorithm treats the various components as a whole rather than individually. In Section 2, we summarize the noise filtering and wavefields separation principles. In Sections 3 and 4, we present the technique using the estimated multicomponent wideband spectral matrices of sources leading to the estimations of the polarization and of the DOA parameters for each wavefield. Finally in Section 5, we present the performances of the algorithm on several simulated 2C-datasets.

\section{Noise Filtering and Wavefield Separation}

In this section, the proposed subspace separation technique based on Multicomponent Wideband Spectral Matrix Filtering (MWSMF) is briefly explained (for more details, the reader might refer to $[14,15])$.

2.1. Model Formulation and Hypothesis. Let us consider an uniform linear array composed of $N_{x}$ omnidirectional sensors uniformly spaced by distance $\Delta$ and receiving $P$ waves with $P<N_{x}$. A convolutive model of seismic signal was first suggested by Robinson [18] and, using the superposition principle, the signal $\mathcal{O}_{i}(t)$ recorded on sensor $i$ is a linear combination of the $P$ waves received on the array added with noise $n_{i}(t)$. Waves have been propagated through a medium and could have been attenuated, time delayed, or phase shifted. The signal $\mathcal{O}_{i}(t)$ recording all wavefieds can be expressed as

with

$$
x_{i}(t)=\sum_{p=1}^{P} a_{p} w_{p}\left(t-\tau_{i}\left(\theta_{p}\right)\right)+n_{i}(t)
$$

(i) $w_{p}(t)$, the waveform signal emitted by a source $p$ (or a wavefied $p$ ),

(ii) $a_{p}$, a random amplitude of the source $p$,

(iii) $\tau_{i}\left(\theta_{p}\right)$, a time propagation between source $p$ and sensor depending of $\theta_{p}$ (the direction-of-arrival (DOA) of source $p$ ),

(iv) $n_{i}(t)$, a random noise supposed to be additive, temporally and spatially white, uncorrelated with the sources, nonpolarized and with a power spectral density given by $\sigma_{n}^{2}$.

In frequential domain, the problem can be divided into a set of instantaneous mixtures of signals as

$$
x_{i}(f)=\sum_{p=1}^{P} a_{p} w_{p}(f) e^{-2 j \pi f \tau_{i}\left(\theta_{p}\right)}+n_{i}(f),
$$

with $x_{i}(f), w_{p}(f)$, and $n(f)$, respectively, the Fourier transform of $x_{i}(t), w_{p}(t)$ and $n_{i}(t)$. The time delay $\tau_{i}\left(\theta_{p}\right)$ can be expressed as summation of two terms

$$
\tau_{i}\left(\theta_{p}\right)=\tau_{0, p}+\xi_{i}\left(\theta_{p}\right),
$$

with $\tau_{0, p}$ the time of propagation between the source and a referenced sensor (classically, the first sensor is used as reference) also called offset. $\xi_{i}\left(\theta_{p}\right)$ is the time of propagation between the reference and sensor $i$ depending of the DOA $\left(\theta_{p}\right)$ of source $p$ as

$$
\xi_{i}\left(\theta_{p}\right)=(i-1) \frac{\Delta \sin \left(\theta_{p}\right)}{V},
$$

where $V$ characterizes the apparent wave velocity and $\Delta$ the distance between two adjacent sensors.

In matrix formulation, (2) can be written as

$$
\underline{X}=\underline{\underline{S}} \underline{A}+\underline{N}
$$

with

(i) $\underline{X}=\left[x_{1}(f), \ldots, x_{i}(f), \ldots, x_{N_{x}}(f)\right]^{T}$, a vector of size $N_{x}$ describing signals recorded on array at frequency bin $f$ ( $T$ stands transposition operator),

(ii) $\underline{\underline{S}}=\left[\underline{S} x_{1}(f), \ldots, \underline{S} x_{p}(f), \ldots, \underline{S} x_{P}(f)\right]$, a matrix of size $N_{x} \times P$ whose columns are steering vectors describing the propagation of each wave with $\underline{S}_{P}(f)=\left[s_{x, 1, p}(f), \ldots, s_{x, N x, p}(f)\right]^{T}$ and $s_{x, i, p}(f)=$ $w_{p}(f) e^{-2 j \pi f \tau_{i}\left(\theta_{p}\right)}$,

(iii) $\underline{A}=\left[a_{1}, \ldots, a_{p}, \ldots, a_{P}\right]^{T}$, a vector of size $P$ which contains the random amplitudes of the waves,

(iv) $\underline{N}$ is a vector of size $N_{x}$ which corresponds to the additive noise.

In case of multicomponent acquisition with vector sensor array, seismic data depend on three parameters: time $\left(N_{t}\right.$ samples), distance ( $N_{x}$ sensors), and number of components ( $N_{c}$ components). These components, recording signals in three directions as $X$ (for in-line axis), $Y$ (for cross-line axis), and $Z$ for vertical axis, allow to express the state of polarization of the different wavefields.

On component $Z$, we can write the recorded signal as

$$
z_{i}(f)=\sum_{p=1}^{P} \alpha_{p} e^{j \varphi_{p}} a_{p} w_{p}(f) e^{-2 j \pi f \tau_{i}\left(\theta_{p}\right)}+n_{i}(f),
$$

where $\alpha_{p}$ and $\varphi_{p}$ are, respectively, the amplitude ratio and the phase-shift between components $X$ and $Z$ characterizing polarization parameters for a source $p$.

In time domain, dataset recorded on a vector array of $N_{x}$ sensors (during $N_{t}$ samples) can be expressed as

$$
\underline{\underline{\underline{T_{t}}}} \in \mathbb{R}^{N_{x} \times N_{t} \times N_{c}} .
$$

In the frequency domain, this dataset is

$$
\underline{\underline{\underline{T}}}=F T\left\{\underline{\underline{\underline{T_{t}}}}\right\} \in \mathbb{C}^{N_{x} \times N_{f} \times N_{c}}
$$


with $N_{f}$ the number of frequency bins. To simplify notations, we will consider the case of 2-component sensors ( $X$ and $Z$ so $N_{c}=2$ ). Nevertheless, proposed method can be used for higher number of components $(3,4,6, \ldots)$. Dataset $T$ is concatenated into a long-vector noted $T$ of size $\left(N_{x} N_{f} \hat{\overline{N_{c}}}\right)$ which contains all frequencies of all sensors and all components:

$$
\underline{T}=\left[\underline{X}\left(f_{1}\right)^{T}, \ldots, \underline{X}\left(f_{N f}\right)^{T}, \underline{Z}\left(f_{1}\right)^{T}, \ldots, \underline{Z}\left(f_{N f}\right)^{T}\right]^{T},
$$

where $\underline{X}\left(f_{i}\right)$ and $\underline{Z}\left(f_{i}\right)$ are vectors of size $N_{x}$ which corresponds to the $i$ th frequency bin received on the $N_{x}$ sensors, respectively, on components $X$ and $Z$. So the mixture model is rewritten as

$$
\underline{T}=\underline{\underline{S}} \underline{A}+\underline{N},
$$

where

(i) $\underline{\underline{S}}=\left[\underline{S}_{1}, \ldots, \underline{S}_{P}\right]$, a matrix of size $N_{x} N_{f} N_{c} \times P$ whose columns are steering vectors describing the propagation of the $P$ waves along the antenna for all frequencies and all components,

(ii) $\underline{A}=\left[a_{1}, \ldots, a_{P}\right]^{T}$, a vector of size $P$ which contains the random amplitudes of the waves,

(iii) $\underline{N}$, a vector of size $N_{x} N_{f} N_{c}$ which corresponds to the additive noises supposed to be additive, temporally and spatially white, uncorrelated with sources, nonpolarized and with identical power spectral density $\sigma_{n}^{2}$.

2.2. Estimation of the Multicomponent Wideband Spectral Matrix. Relations between components and sensors are expressed in the calculation of the Multicomponent Wideband Spectral Matrix (MWSM) $\underline{\underline{\Gamma}}$ of $\underline{T}$ as

$$
\widehat{\underline{\Gamma}}=E\left[\underline{T} \underline{T}^{H}\right]
$$

with $E$ the expectation operator and $H$ the transpose conjugate operation.

To avoid the fact that $\underline{T} \underline{T}^{H}$ is noninvertible (not full rank) and to decorrelate sources from noise and from themselves, we perform smoothing operators to estimate the matrix $\hat{\underline{\Gamma}}$. This step is crucial since the effectiveness of filtering depends on this estimation. In practice, mathematical expectation operator $E$ is an averaging operation, like spatial or frequential smoothing or both of them [19-22]. Objective of these averaging operations is to reduce the influence of terms corresponding to the interactions between different sources in order to uncorrelate them, and to uncorrelate sources and noise, making the inversion of the spectral matrix possible. The spatial smoothing could be done by averaging spatial sub-bands. The uniform linear array with $N_{x}$ sensors is subdivided into overlapping subarrays in order to have several identical arrays, which will be used to estimate spectral matrices in order to build a smoothed matrix. Shan et al. [21] have proven that if the number of subarrays is greater than or equal to the number of sources $\mathrm{Nw}$, then the spectral matrix of the sources is nonsingular. However, one assumption is that the wave does not vary rapidly over the number of sensors used in the average, in particular, amplitude fluctuations must be smoothed out. To introduce frequency smoothing, two ways can be performed: either by weighting the autocorrelation and cross-correlation functions (in the time domain) or by averaging frequential subbands (in the frequential domain). For a better estimation of the multicomponent wideband spectral matrix, it is suitable to realize jointly spatial and frequential smoothing. For more details on averaging operators, we suggest to read [14].

The multicomponent wideband spectral matrix $\widehat{\underline{\Gamma}}$ is a matrix of dimension $N_{x} N_{f} N_{c} \times N_{x} N_{f} N_{c}$. The structure of $\underline{\underline{\underline{\Gamma}}}$ is presented diagrammatically on Figure $1 . \widehat{\underline{\Gamma}}_{X, X}$ and $\widehat{\underline{\Gamma}}_{Z, Z}$ correspond to the single-component wideband spectral matrix for $X$ and $Z$ components, respectively. These terms are located on the main diagonal of $\underline{\underline{\Gamma}}$. The other blocks correspond to the cross-component spectral matrices which contain information relating to the interaction between the components and especially information on polarization. The results obtained by multicomponent wideband matrix filtering are better than the ones obtained applying classical filtering methods on each components for the reason that the former contains more information on the signal especially on polarization Since the multicomponent wideband matrix filtering provides more information on the signal and especially on polarization, better filtering results are obtained rather than results based on classical filtering methods used independently on each component.

2.3. Estimation of Signal Subspace. Following the assumptions made in Section 2.1, $\underset{\underline{\Gamma}}{\underline{\Gamma}}$ can be written as

$$
\underline{\underline{\Gamma}}=\underline{\underline{S}} \underline{\underline{\underline{\Gamma}}}_{A} \underline{\underline{S}}^{H}+\sigma_{n}^{2} \underline{\underline{I}} .
$$

After smoothing (averaging) operators step, $\widehat{\underline{\underline{\Gamma}}}_{A}=$ $E\left[\begin{array}{ll}\underline{A} & \underline{A}^{H}\end{array}\right]$ is nonsingular (with full rank equals to $P$ in case of free noise dataset or equals to $N-x$ in case of noisy dataset). As columns of $\underline{\underline{S}}$ are linearly independent, then the rank of the signal part $\underline{\underline{S}} \underline{\underline{\Gamma}}_{A} \underline{\underline{S}}^{H}$ is $P$. So the estimated spectral matrix can be decomposed uniquely using an eigenvalue decomposition as

$$
\widehat{\underline{\Gamma}}=\sum_{i=1}^{N_{x} N_{f} N_{c}} \lambda_{i} \underline{u}_{i} \underline{u}_{i}^{H}
$$

where $\lambda_{i}$ and $\underline{u}_{i}$ are, respectively, the real eigenvalues, the orthonormal eigenvectors of $\hat{\underline{\Gamma}}$. Eigenvalues can be arranged in decreasing order $\left(\lambda_{1} \geq \overline{\lambda_{2}} \geq \cdots \geq \lambda_{N_{x} N_{f} N_{c}} \geq \sigma_{n}^{2}\right.$. $)$. Each eigenvalue $\lambda_{i}$ corresponds to the energy of the data associated with their respective eigenvector $\underline{u}_{i}$. The space generated by the smallest eigenvectors associated to the smallest eigenvalues is referred to as the noise subspace $\widehat{\underline{\underline{T}}}_{N}$, and its orthogonal complement as the signal subspace $\widehat{\underline{\underline{T}}}_{S}$, spanned by the steering vectors of the signal [23]. Estimated 


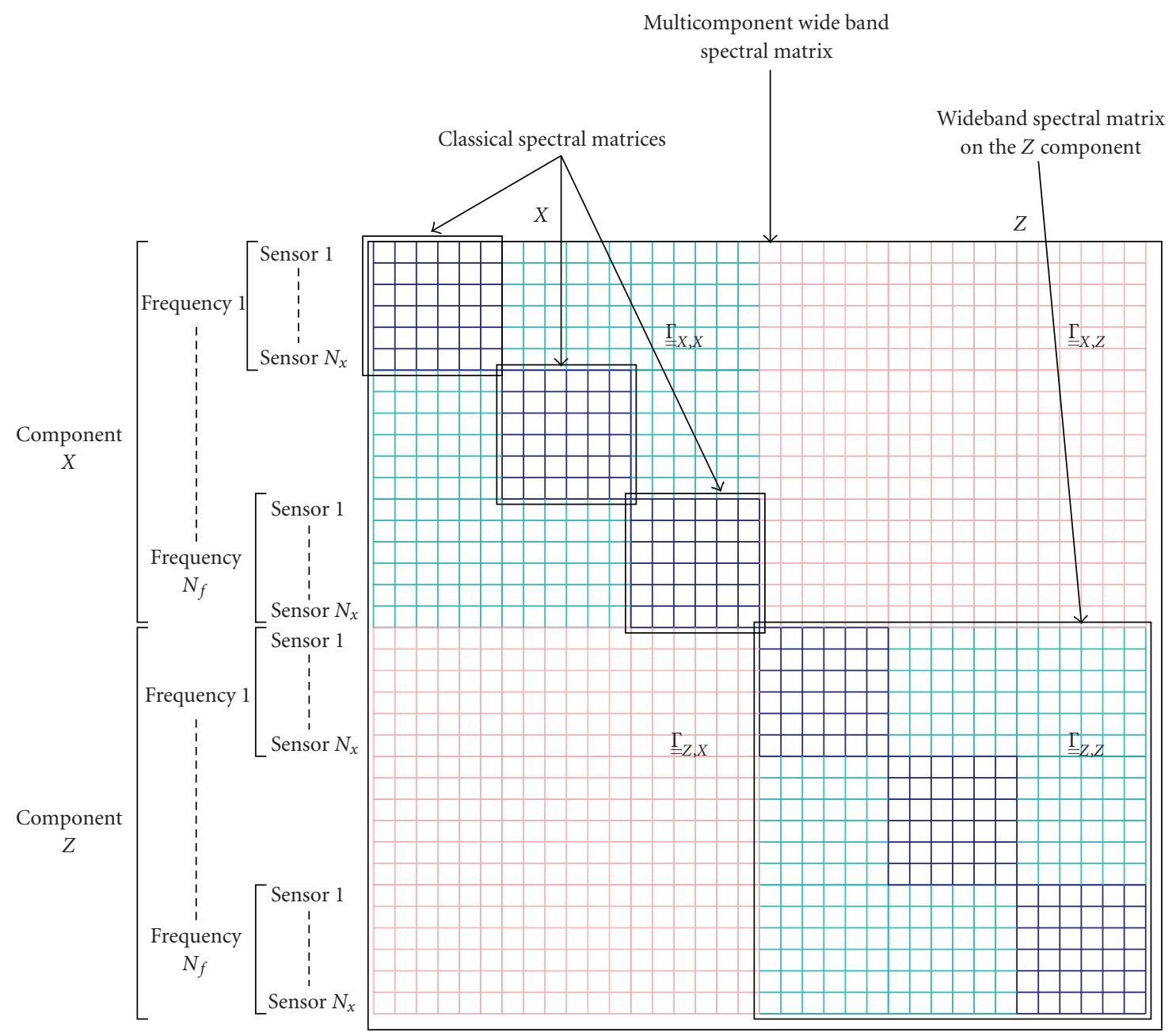

FIGURE 1: Diagram of a 2-Component wideband spectral matrix showing in red the cross-component matrices, in blue the inner-component matrices, and in black each classical spectral matrices defined at each frequency bin.

Multicomponent Wideband Spectral Matrix can be written as

$$
\widehat{\underline{\Gamma}}=\underline{\underline{\Gamma}}_{S}+\underline{\underline{\Gamma}}_{N}=\sum_{i=1}^{P} \lambda_{i} \underline{u}_{i} \underline{u}_{i}^{H}+\sum_{k=P+1}^{N_{x} N_{f} N_{c}} \lambda_{k} \underline{u}_{k} \underline{u}_{k}^{H} .
$$

After performing efficient average, a decorrelation of waves from themselves and waves from noise is obtained and the spectral matrix is well estimated. Under these conditions, Thirion et al. in [24-26] have shown that the steering vectors are identifiable to the eigenvectors. In fact steering vectors that account several frequencies (wideband context) can easily show to be asymptotically orthogonal. In that case, the spectral matrix corresponding to the pth source (wave) is noted $\underline{\underline{\Gamma}}_{s, p}$ and is equal to

$$
\widehat{\underline{\Gamma}}_{s, p}=\lambda_{p} \underline{u}_{p} \underline{u}_{p}^{H} .
$$

2.4. Filtering by Projection onto the Signal Subspace. The filtering step corresponds to an orthogonal projection of the initial data $\underline{T}$ onto the first $P$ eigenvectors corresponding to the signal subspace:

$$
\underline{T}_{s}=\sum_{i=1}^{P}\left\langle\underline{T}, \underline{u}_{i}\right\rangle \underline{u}_{i} .
$$

The projection onto the noise subspace $\left(\underline{T}_{n}\right)$ is obtained by subtraction of $\underline{T}_{s}$ from the initial data

$$
\underline{T}_{n}=\underline{T}-\underline{T}_{s}=\sum_{i=P+1}^{N_{x} N_{f} N_{c}}\left\langle\underline{T}, \underline{u}_{i}\right\rangle \underline{u}_{i} .
$$

The final steps consist of rearranging the long-vectors $\underline{T}_{s}$ and $\underline{T}_{n}$ in its initial form and computing an inverse Fourier transform in order to come back to the time-distancecomponent domain.

\section{Polarization Estimation}

3.1. Introduction. After presenting the separation processing part, we propose to find polarization parameters on 


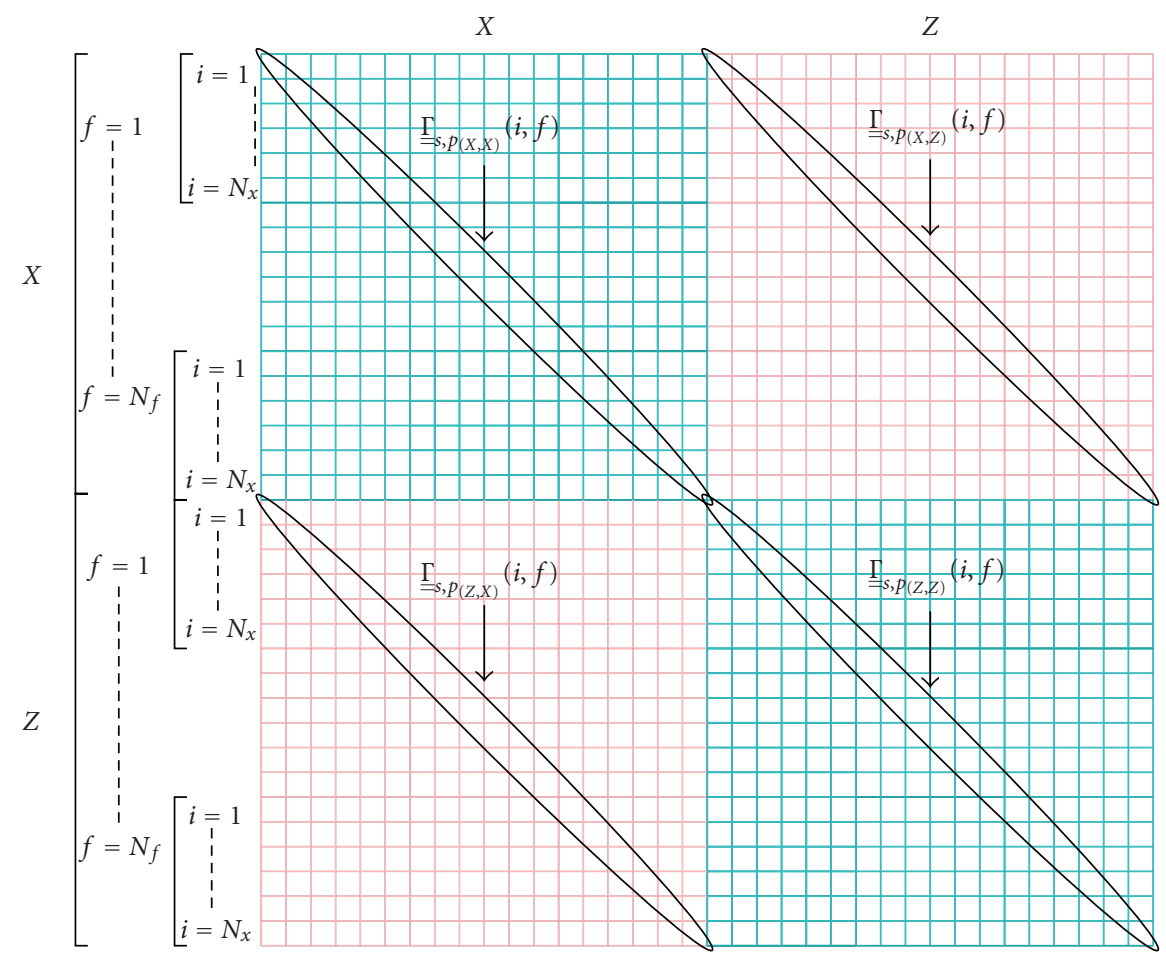

FIgURE 2: Diagram of $\underline{\underline{\Gamma}}_{s, p}$, the 2-Component wideband spectral matrix of the wave $p$.

each separated wavefield. State of polarization analysis is based on the computation of parameters describing the particle movement associated with wave propagation. That movement of the ground induced useful parameters which were first identified by Jolly in 1956 [27], whereas the first attempt to measure this movement was done by Shimshoni and Smith in 1964 [28]. They introduced a successful method of polarization analysis for earthquake data. Many other algorithms were developed subsequently for seismic exploration applications [29-31]. One of the most effective and stable approaches in this regards is the algorithm developed by Flinn $[32,33]$ using the covariance matrix of the data. In the following part, we compare our proposed method (based on MWSM) with Flinn's algorithm.

3.2. Proposed Method. We propose to use the spectral matrices of rank one (linked to each pth source) obtained from the decomposition of the multicomponent wideband spectral matrix (see (15)). Thus, once a wavefield has been separated from other waves and from noise, we show that its polarization parameters can be characterized from the matrix elements of wavefield, $\widehat{\underline{\underline{\Gamma}}}_{s, p}$. After separation processing, the signal, noted $\mathcal{O}_{x, p, i}(f)$, corresponding to the $p$ th source received on component $X$ at frequency $f$ and on sensor $i$ can be expressed as

$$
\mathcal{O}_{x, p, i}(f)=a_{p} w_{p}(f) e^{-j 2 \pi f \tau_{i}\left(\theta_{p}\right)},
$$

where $\tau_{i}\left(\theta_{p}\right)$ is the time of propagation between the source and the sensor $i$ (for wave $p$ ). For the second component $Z$, we obtain

$$
\mathcal{O}_{z, p, i}(f)=\alpha_{p} e^{j \varphi_{p}} a_{p} w_{p}(f) e^{-2 j \pi f \tau_{i}\left(\theta_{p}\right)}
$$

The diagonal element of $\underline{\underline{\underline{T}}}_{s, p}$ at the frequency $f$ on the $i$ th sensor which corresponds to the interaction of the $X$ component with itself could be expressed by

$$
\widehat{\underline{\Gamma}}_{s, P(X, X)}(i, f)=\sigma_{p}^{2}\left|w_{p}(f)\right|^{2} .
$$

Likewise, the term corresponding to the interaction of $\mathrm{Z}$ component with itself is

$$
\widehat{\underline{\Gamma}}_{s, p(Z, Z)}(i, f)=\alpha_{p}^{2} \sigma_{p}^{2}\left|w_{p}(f)\right|^{2}
$$

and finally, the cross-term corresponding to the interaction between component $X$ and $Z$ could be written as

$$
\widehat{\underline{\Gamma}}_{s, p_{(X, Z)}}(i, f)=\alpha_{p} \sigma_{p}^{2}\left|w_{p}(f)\right|^{2} e^{-j \varphi_{p}}
$$

All these terms are located either on the principal diagonal or on the secondary diagonals of the matrix $\widehat{\Gamma}_{s, p}$ (see Figure 2). Based on these structures, we deduce estimators for polarization parameters of the $p$ th wave on each sensor $i$ at frequency $f$. In fact, polarization parameters $\left(\alpha_{p}, \varphi_{p}\right)$ 


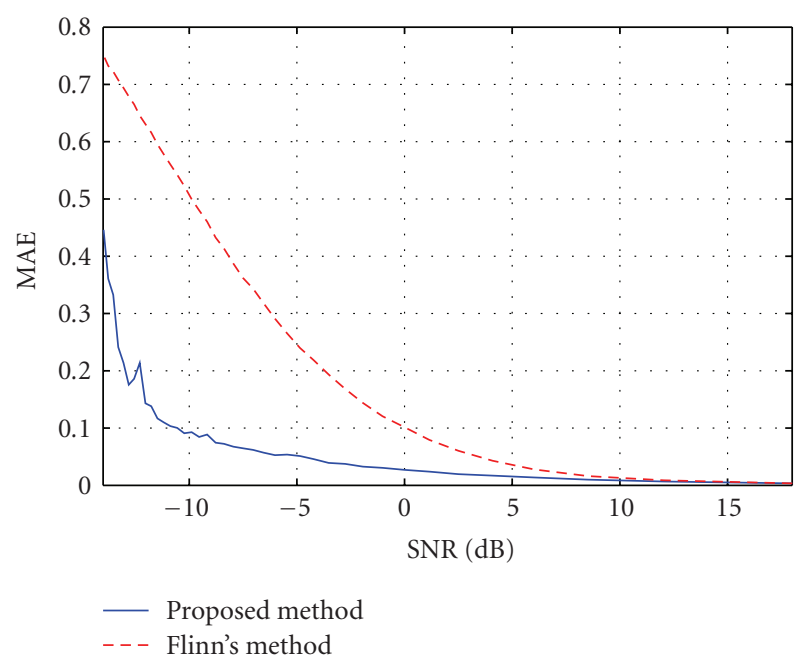

Figure 3: Mean Absolute Error for estimation of phase shift between components for various SNRs (plain line: MWSM based method; dotted line: Flinn's method).

expressed as amplitude ratio between components $X$ and $Z$ and phase shift for wave $p$ can be expressed, respectively, by

$$
\begin{gathered}
\widehat{\alpha_{p(X, Z)}}(i, f)=\sqrt{\frac{\widehat{\underline{\underline{\Gamma}}}_{s, p(Z, Z)}(i, f)}{\widehat{\underline{\underline{\Gamma}}}_{s, p_{(X, X)}}(i, f)},} \\
\widehat{\varphi_{p_{(X, Z)}}}(i, f)=\arg \left[\widehat{\underline{\underline{\Gamma}}}_{s, p_{(X, Z)}}(i, f)\right] .
\end{gathered}
$$

Classically, the propagation medium is regarded as isotropic (nondispersive for frequency) so that the polarization parameters are independent of frequency and sensor. But in more realistic context where some dispersion appears, a better estimate of the polarization parameters can thus be obtained by averaging them over a range of frequencies and sensors. However, in order to have a correct estimate, the averaging must be done only on the frequencies belonging to the signal bandwidth $\left(L_{3 \mathrm{~dB}}=\left[f_{\text {inf }}, f_{\text {sup }}\right]\right)$. The estimators thus obtained are

$$
\begin{gathered}
\widehat{\alpha_{p(X, Z)}}=\sqrt{\frac{1}{\operatorname{card}\left(L_{3 \mathrm{~dB}}\right) \cdot N_{x}} \sum_{i=1}^{N_{x}} \sum_{f=f_{\text {inf }}}^{f_{\text {sup }}} \frac{\widehat{\underline{\Gamma}}_{s, p_{(Z, Z)}}(i, f)}{\widehat{\underline{\Gamma}}_{s, p_{(X, X)}}(i, f)},} \\
\widehat{\varphi_{p(X, Z)}}=\arg \left[\frac{1}{\operatorname{card}\left(L_{3 \mathrm{~dB}}\right) \cdot N_{x}} \sum_{i=1}^{N_{x}} \sum_{f=f_{\text {inf }}}^{f_{\text {sup }}} \underline{\underline{\Gamma}}_{s, p_{(X, Z)}}(i, f)\right]
\end{gathered}
$$

with $\operatorname{card}\left(L_{3 \mathrm{~dB}}\right)$ being the cardinal of $L_{3 \mathrm{~dB}}$.

\subsection{Comparison between Flinn's Method and Proposed Method} Based on MWSM. MWSM's and Flinn's methods are two different approaches for polarization analysis. Flinn's method uses a covariance matrix and proposes a temporal approach on a single trace whereas our proposed method is a frequential approach which can either be on a single trace or on the whole array. In the case studied here, the waves'

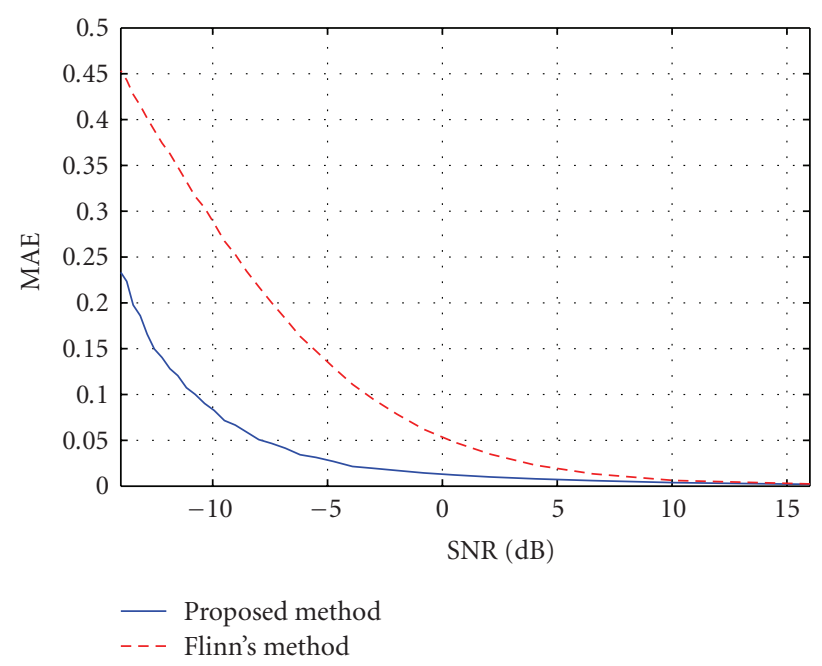

Figure 4: Mean Absolute Error for estimation of the amplitude ratio between components for various SNRs (plain line: MWSM based method; dotted line: Flinn's method).

state of polarization can be considered as constant over distance. Consequently, for a given wave, we can estimate the amplitude ratio and phase shift between the components by carrying out an averaging of the parameters found on each sensor.

To compare Flinn's and our method and to illustrate polarization estimation, we consider the trivial case of a single wave with infinite velocity, received on a $2 \mathrm{C}$-sensors array, whose phase shift $\varphi(=0.4 \mathrm{rad})$ and amplitude ratio $\alpha$ $(=0.8)$ are constant over the array.

Figures 3 and 4 correspond respectively to the Mean Absolute Error (MAE) between the theoretical and the estimated values of phase shift and amplitude ratio for various signal-to-noise ratio (SNR) from $-10 \mathrm{~dB}$ to $15 \mathrm{~dB}$. The average is done for 500 noisy realizations.

These figures show that for both methods, polarization analysis is very sensitive to noise and thus the estimates are better when SNR increases. We can notice that our MWSMbased method always gives better estimation than the Flinn's method for small SNR.

\section{Direction-of-Arrival Estimation}

4.1. Proposed Method. Just as we did for polarization state estimation, we propose a DOA estimation method based on the structure of Multicomponent Wideband Spectral Matrix. We call it MW-MUSIC for Multicomponent WidebandMUSIC as it is an extension of the MUSIC (MUltiple SIgnal Classification) algorithm [34-37]. This method is an extension of the MUSIC algorithm for vector-sensor arrays called LV-MUSIC for Long-Vector MUSIC [1]. The first extensions of MUSIC algorithm to polarized sources were made by Schmidt [34], Ferrara and Parks [38], Wong and Zoltowski [6, 8], and Wong et al. [13]. Algorithms to estimate DOAs of polarized sources in electromagnetism were also proposed in $[1,39,40]$. Our proposed method has the advantage of being able to compute both DOA and offset. 


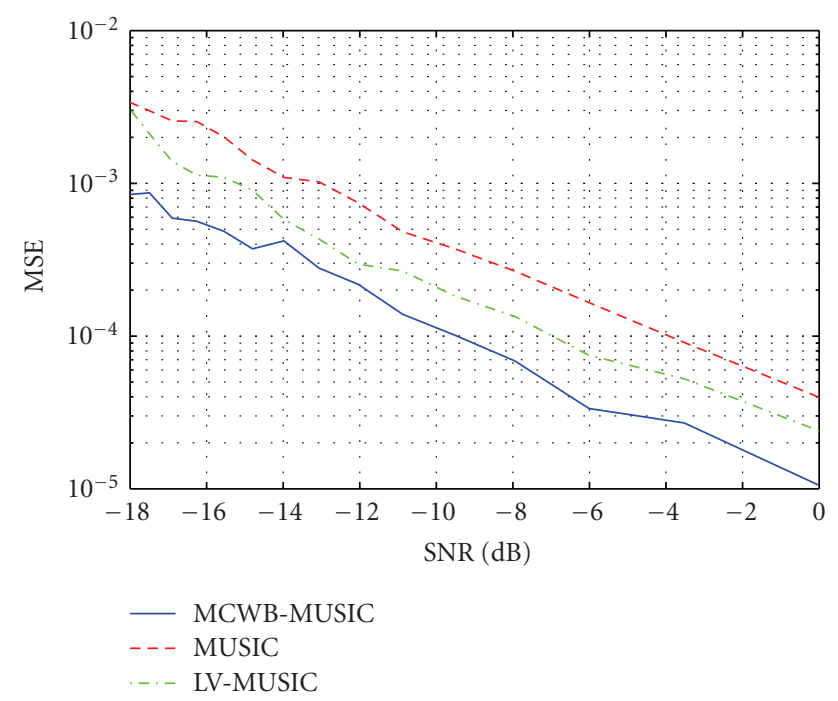

FIgURE 5: Comparison of DOA estimation error for various methods. (dotted line: Classical MUSIC; semidotted line: LVMUSIC; plain line: MW-MUSIC).

We define two matrices $\underline{\underline{U}}_{s}\left(N_{x} N_{f} N_{c} \times P\right.$ size $)$ and $\underline{\underline{U}}_{n}$ $\left(N_{x} N_{f} N_{c} \times\left(N_{x} N_{f} N_{c}-P\right)\right.$ size $)$, containing the eigenvectors corresponding to signal subspace and noise subspace, respectively,

$$
\begin{gathered}
\underline{\underline{U}}_{s}=\left[\underline{u}_{1}, \ldots, \underline{u}_{P}\right], \\
\underline{\underline{U}}_{n}=\left[\underline{u}_{P+1}, \ldots, \underline{u}_{N_{x} N_{f} N_{c}}\right] .
\end{gathered}
$$

These complex matrices enable us to write MWSM as

$$
\underline{\underline{\Gamma}}=\underline{\underline{U}}_{s} \underline{\underline{\Lambda}}_{s} \underline{\underline{U}}_{s}^{H}+\sigma_{b}^{2} \underline{\underline{U}}_{n} \underline{\underline{U}}_{n}^{H}
$$

with $\Lambda_{s}$ being a diagonal matrix containing the $P$ highest eigenvalues. If we multiply (12) on the right by $\underline{\underline{U}}_{n}$, we obtain

$$
\underline{\underline{\Gamma}} \underline{\underline{U}}_{n}=\underline{\underline{S}} \underline{\underline{\Gamma}}_{A} \underline{\underline{S}}^{H} \underline{\underline{U}}_{n}+\sigma_{b}^{2} \underline{\underline{U}}_{n} .
$$

By combining (27) and (28) and by using the orthogonality property of the matrices $\underline{\underline{U}}_{s}$ and $\underline{\underline{U}}_{n}$, we obtain

$$
\sigma_{b}^{2} \underline{\underline{U}}_{n}=\underline{\underline{S}}_{\underline{\underline{\Gamma}}} \underline{\underline{S}}^{H} \underline{\underline{U}}_{n}+\sigma_{b}^{2} \underline{\underline{U}}_{n}
$$

which implies:

$$
\underline{\underline{S}}^{H} \underline{\underline{U}}_{n}=\underline{\underline{0}}
$$

We can rewrite it as

$$
\underline{S}_{p}^{H} \underline{\underline{U}}_{n} \underline{\underline{U}}_{n}^{H} \underline{S}_{p}=0
$$

with $\underline{S}_{p}$, being the propagation vector corresponding to the wave $p$. Thereafter, we note $\underline{\underline{\Pi}}_{n}=\underline{\underline{U}}_{n} \underline{\underline{U}}_{n}^{H}$, the matrix corresponding to the projection on noise subspace.

According to relation (31), propagation vectors are orthogonal to noise subspace. Consequently, their projection on $\underline{\underline{\Pi}}_{n}$ is zero. MW-MUSIC algorithm exploits this idea by

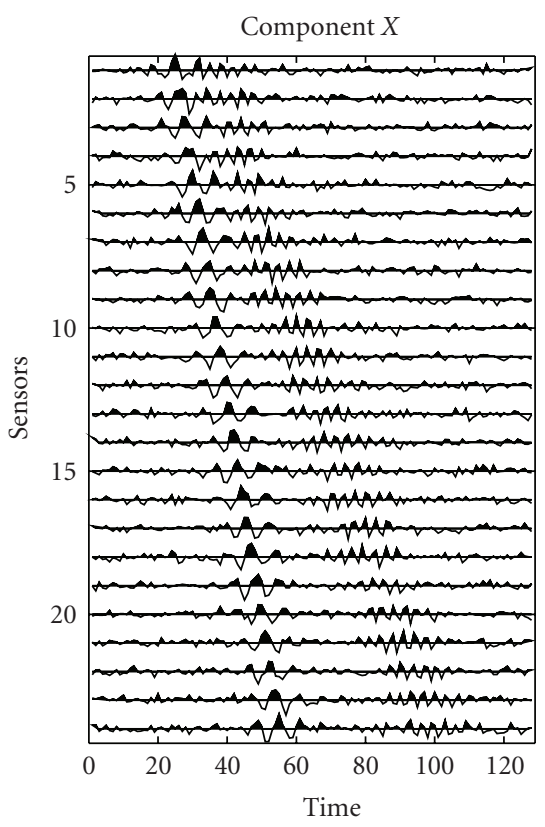

(a)

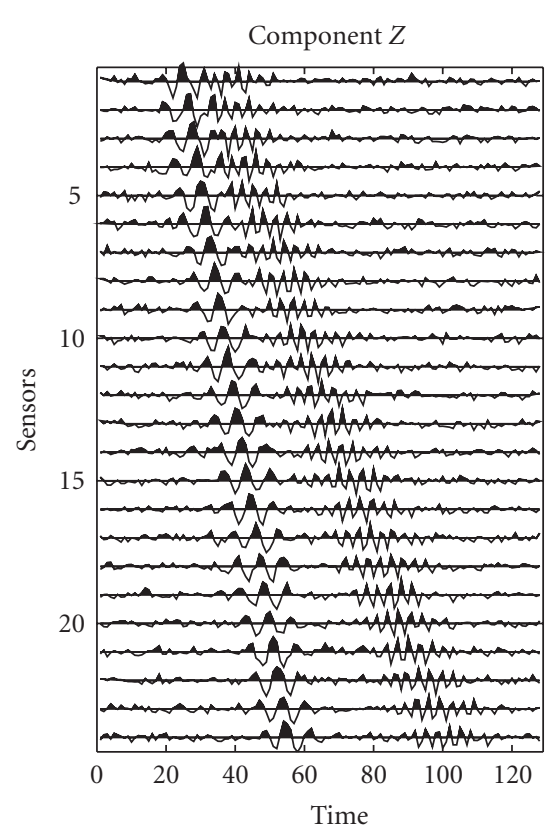

(b)

Figure 6: 2-Component initial dataset in time and distance ((a): Horizontal component $X$, (b): Vertical component $Z$ ).

carrying out the projection of directional vector $\underline{h}\left(\theta, \tau_{0}, \alpha, \varphi\right)$ on the estimated noise subspace. This vector models the arrival of a polarized wave of direction $\theta$ on multicomponent sensors' antenna and is expressed as

$$
\underline{h}\left(\theta, \tau_{0}, \alpha, \varphi\right)=\frac{1}{N_{x} N_{f} N_{c}}\left(\begin{array}{c}
\frac{d}{\alpha e^{j \varphi}}\left(\theta, \tau_{0}\right) \\
\left(\theta, \tau_{0}\right)
\end{array}\right)
$$




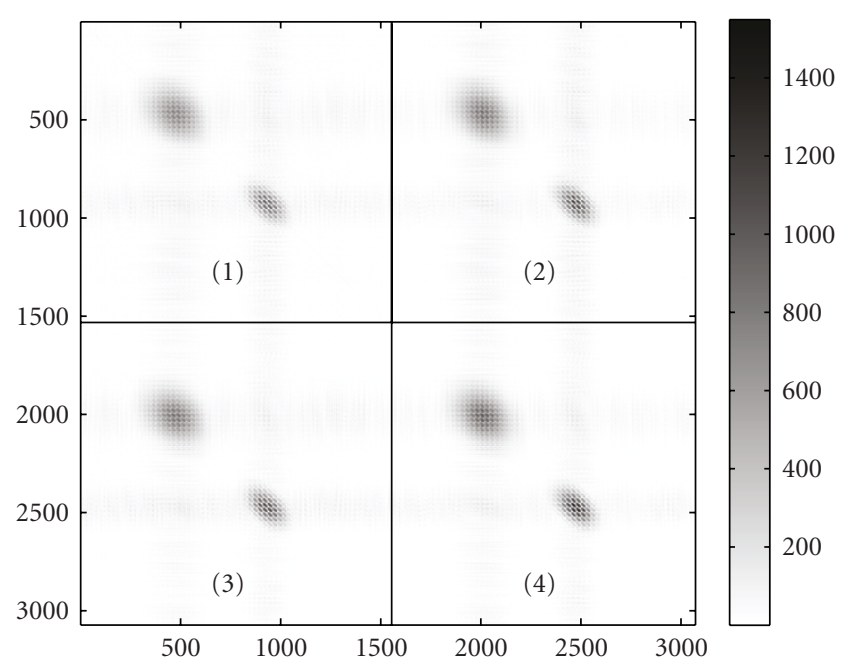

Figure 7: Modulus of the Multicomponent Wideband Spectral Matrix of the initial dataset (of size $3072 \times 3072$ with $3072=$ $24 \times 64 \times 2$ ). Compared with Figure 1, block 1 is $\underline{\underline{\Gamma}}_{X X}$, block 4 is $\underline{\underline{\Gamma}}_{Z Z}$ and blocks 2 and 3 are $\underline{\underline{\Gamma}}_{X Z}, \underline{\underline{\Gamma}}_{Z X}$, respectively.

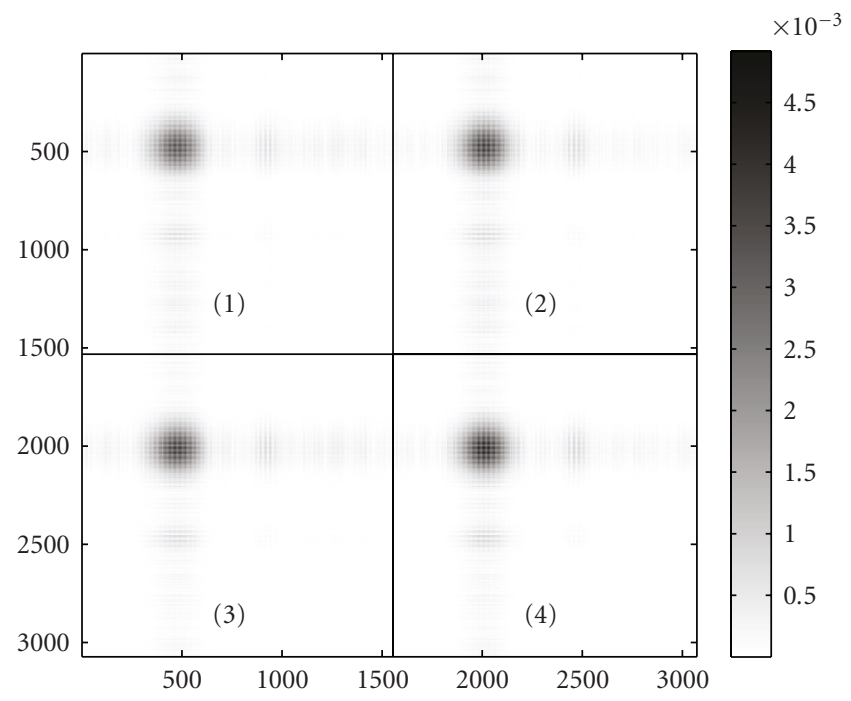

Figure 8: Modulus of the multicomponent wideband spectral matrix of the first extracted wave, $\underline{\underline{\Gamma}}_{s, 1}$.

with

$$
\begin{gathered}
\underline{d}\left(\theta, \tau_{0}\right)=\left(\begin{array}{c}
w\left(f_{1}\right) e^{-j 2 \pi f_{1} \tau_{0}} \underline{e}\left(\theta, f_{1}\right)^{T} \\
\vdots \\
w\left(f_{N f}\right) e^{-j 2 \pi f_{N f} \tau_{0}} \underline{e}\left(\theta, f_{N f}\right)^{T}
\end{array}\right), \\
\underline{e}\left(\theta, f_{n}\right)=\left(\begin{array}{c}
1 \\
\vdots \\
e^{-j 2 \pi f_{n}(i-1)(\Delta \sin (\theta) / V)} \\
\vdots \\
e^{-j 2 \pi f_{n}\left(N_{x}-1\right)(\Delta \sin (\theta) / V)}
\end{array}\right)
\end{gathered}
$$

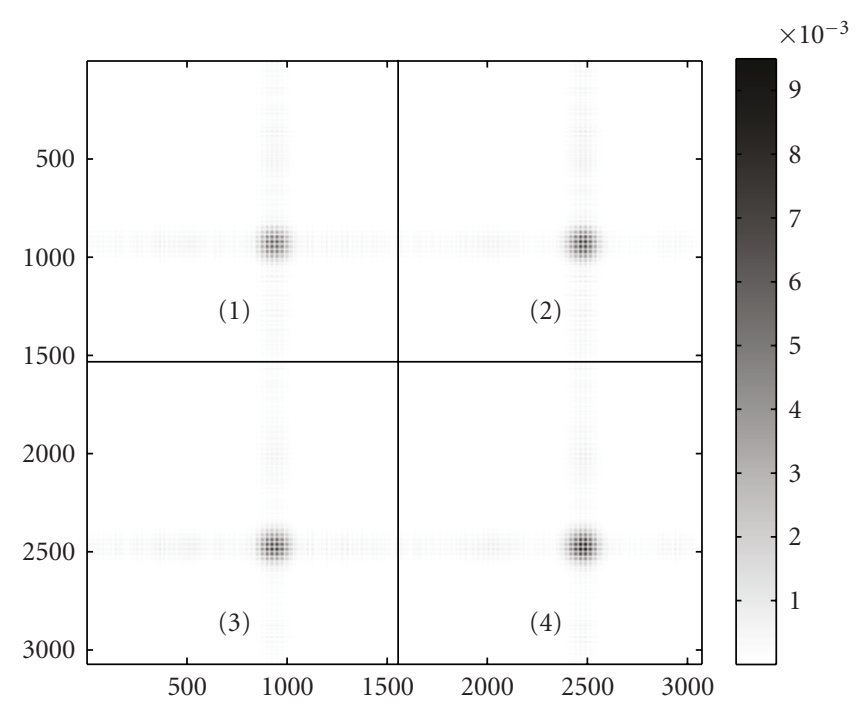

Figure 9: Modulus of the multicomponent wideband spectral matrix of the second extracted wave $\underline{\underline{\Gamma}}_{s, 2}$.

The extended MUSIC functional, calculated by projection of $\underline{h}\left(\theta, \tau_{0}, \alpha, \varphi\right)$ on the noise subspace is given by

$$
\begin{aligned}
\operatorname{MW} & -\operatorname{MUSIC}\left(\theta, \tau_{0}, \alpha, \varphi\right) \\
& =\frac{1}{\underline{h}\left(\theta, \tau_{0}, \alpha, \varphi\right)^{H} \underline{\Pi}_{n} \underline{h}\left(\theta, \tau_{0}, \alpha, \varphi\right)} .
\end{aligned}
$$

The functional allows local maxima for a set of values $\theta, \tau_{0}, \alpha$, and $\varphi$. We use the fact that two parameters $(\alpha, \varphi)$ have been already found by the method proposed on Section 3. After this stage, the functional only depends on two parameters, $\theta$ the direction of arrival and $\tau_{0}$, the offset, and it will give maximum for values of $\left(\theta, \tau_{0}\right)$ corresponding to the sources present in the signal. It is clear that processing will work with lot of efficiency in case of far field sources (waves can be considered as locally plane).

4.2. Study of Estimator Variance. In this part, we compare variances of various estimators: MUSIC, LV-MUSIC and MW-MUSIC. Mean Square Error (MSE) for DOA estimation is presented for various SNRs (Figure 5). Each point corresponds to an average over 200 realizations. In this case under study, a polarized source is received on an array of $302 \mathrm{C}$-sensors. The DOA of the source, expressed in terms of samples of delay between two sensors, is 1 sample. A white Gaussian noise is added to the signal with a SNR from $-18 \mathrm{~dB}$ to $0 \mathrm{~dB}$. For classical MUSIC algorithm which operates only on one component, we carry out an average of the results obtained from the two components. Figure 5 shows clearly that when we take into account the polarization information and frequential coherency given by the MWSM's structure, statistical performances of the estimator are improved. This induces that MW-MUSIC gives better results rather than Classical-MUSIC algorithm. 


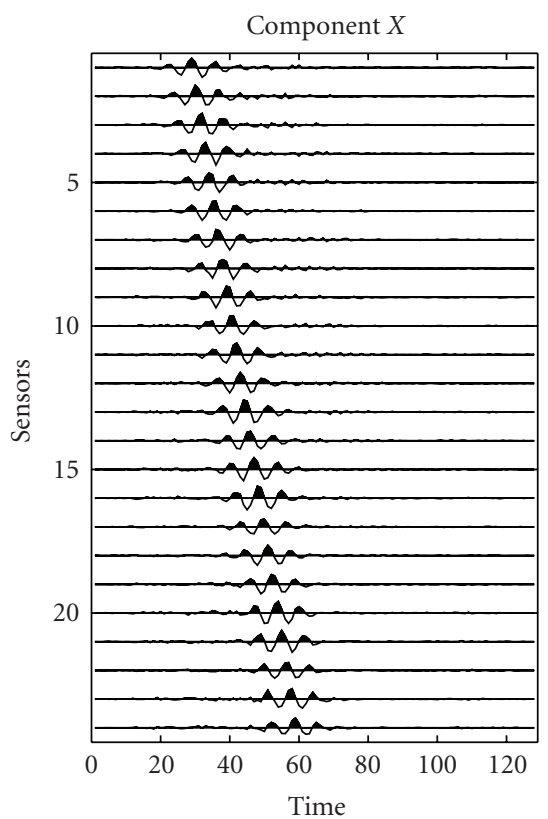

(a)

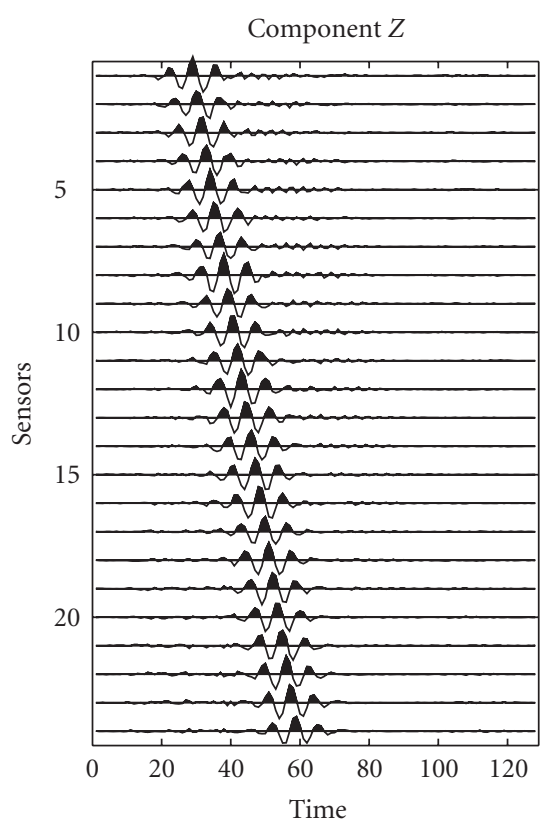

(b)

Figure 10: First extracted wave after projection of initial dataset onto the first eigenvector. (a) Component $X$, (b) Component $Z$.

\section{Synthetic Examples}

Proposed method consisting of wavefield separation followed by polarization and DOA estimation steps is applied on a 2-Components synthetic seismic profile to validate the efficiency of the method. Figure 6 plots two waves received on an array of $242 \mathrm{C}$-sensors superimposed by a white gaussian noise $(\mathrm{SNR}=4 \mathrm{~dB})$. The fastest wave, $($ called wave 1$)$ has a linear polarization parameter $(\alpha=1.5$ and $\varphi=0 \mathrm{rad})$

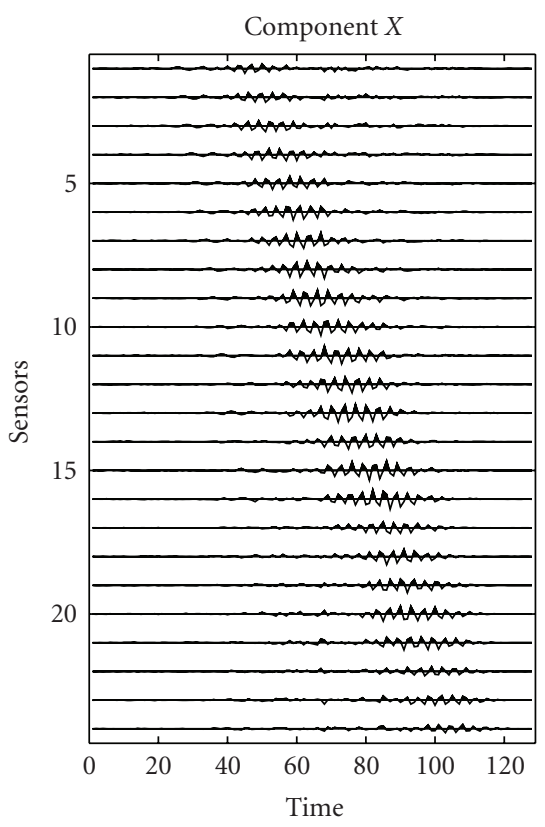

(a)

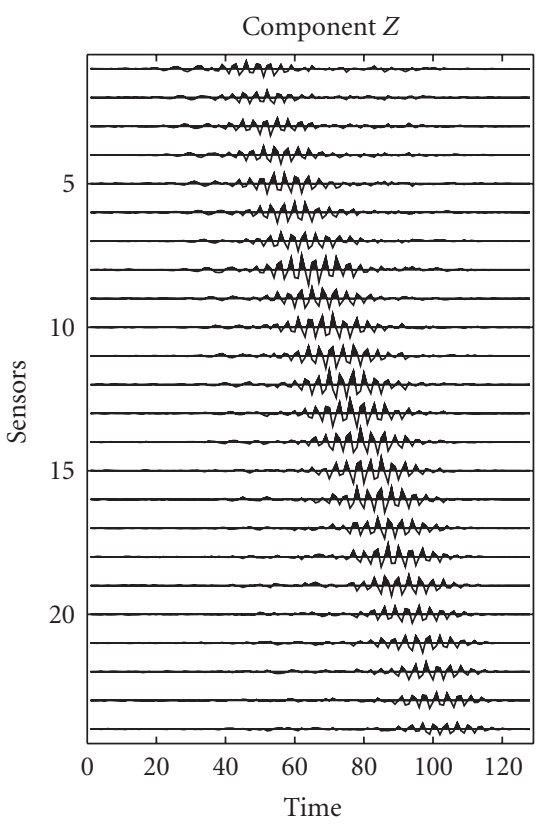

(b)

FIGURE 11: Second extracted wave after projecting initial dataset on the second eigenvector. (a) Component $X$, (b) Component $Z$.

and the slowest wave (called wave 2 ) is characterized by an elliptic polarization $(\alpha=1.5$ and $\varphi=1.5 \mathrm{rad})$.

5.1. Wavefield Separation. The first step of proposed method consists of separating the two waves and the noise using the multicomponent wideband spectral matrix filtering technique (see Section 2). Estimation of the Multicomponent Wideband Spectral Matrix (MWSM) is done using both spatial and frequential smoothing. Its modulus of this matrix 


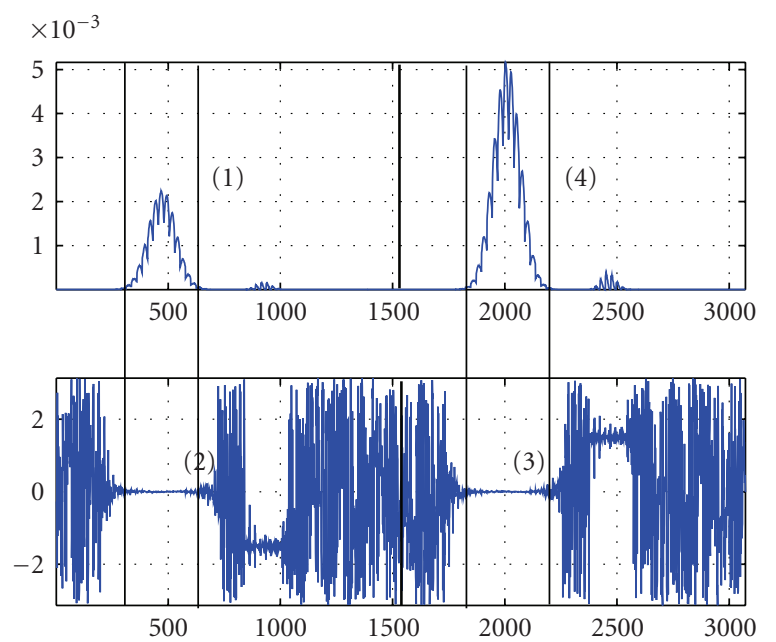

FIGURE 12: Upper plot $=$ modulus of diagonals of blocks (1) and (4) diagonals of the spectral matrix of wave 1 (Figure 8). Bottom plot: angle of blocks (2) and (3) diagonals of the spectral matrix of wave 1 (Figure 8).

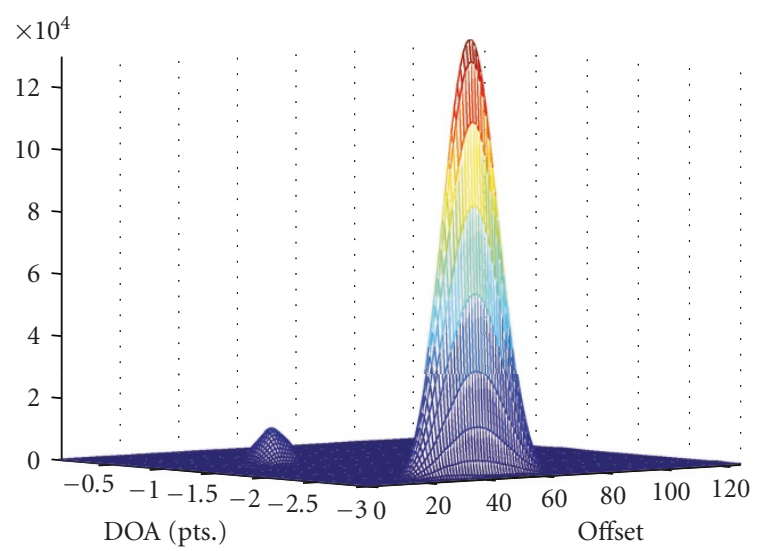

FIgUre 13: Amplitude of MW-MUSIC functional as a function of DOA and offset.

is presented in Figure 7. We observe the same structure as in Figure 1 where the large energetic pattern is associated to wave 1 (low frequency content), and smaller energetic pattern to wave 2 (high frequency content). The MWS Matrix is then decomposed using eigenvalue decomposition. Observing the decrease of eigenvalues, we decide to keep the two first eigen sections, corresponding to modeled waves:

$$
\underline{\underline{\Gamma}}_{s, 1}=\lambda_{1} \underline{u}_{1} \underline{u}_{1}^{H}, \quad \underline{\underline{\Gamma}}_{s, 2}=\lambda_{2} \underline{u}_{2} \underline{u}_{2}^{H} .
$$

Figures 8 and 9 show the modulus of $\underline{\underline{\Gamma}}_{s, 1}$ (wave 1) and $\underline{\underline{\Gamma}}_{s, 2}$ (wave 2). We can observe from these figures that separation of waves is efficient since patterns are well separated and no energetic interferences appear. Thus, by projecting the initial data on the first eigenvector $\underline{u}_{1}$, we obtain the first extracted wavefield (wave 1) (Figure 10) and on the second eigenvector $\underline{u}_{2}$, the second extracted wavefield (wave 2) (Figure 11). These two figures clearly show that the noise has been removed and the waves have been well separated.

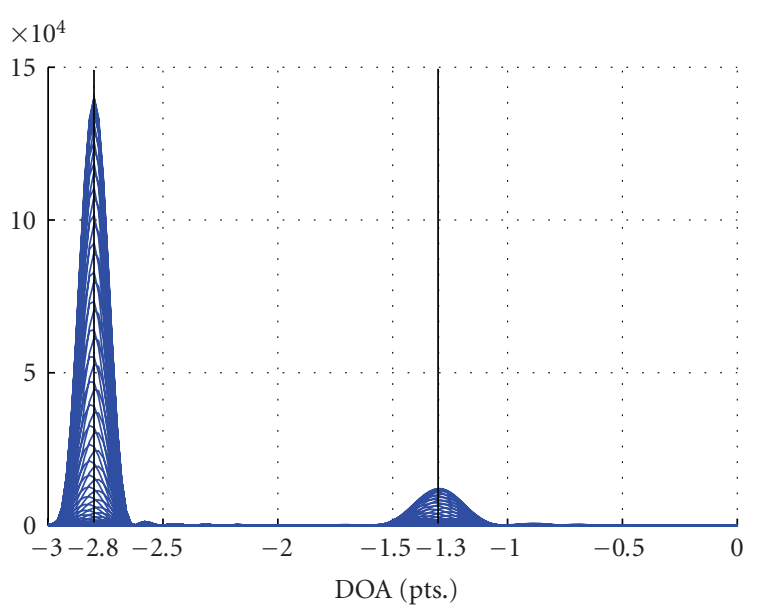

FIGURE 14: DOA estimation (cross-section for various offsets).

TABle 1: True and estimated values of polarization parameters of wave 1 and wave 2 .

\begin{tabular}{ccc}
\hline & True values & Estimated values \\
\hline wave 1 & $\alpha_{1}=1.5$ & $\widehat{\alpha_{1}}=1.1$ \\
& $\varphi_{1}=0 \mathrm{rad}$ & $\widehat{\varphi_{1}}=3 \cdot 10^{-2} \mathrm{rad}$ \\
\hline \multirow{2}{*}{ wave 2 } & $\alpha_{2}=1.5$ & $\widehat{\alpha_{2}}=1.17$ \\
& $\varphi_{2}=1.5 \mathrm{rad}$ & $\widehat{\varphi_{2}}=1.54 \mathrm{rad}$ \\
\hline
\end{tabular}

5.2. Polarization Estimation. After separation of waves, the second step consists of making the polarization analysis of the two waves using the matrix elements of $\underline{\underline{\Gamma}}_{s, 1}$ and $\underline{\underline{\Gamma}}_{s, 2}$ (see Section 3 ). As previously presented, in Figure 8 (resp., Figure 9), blocks (1) and (4) correspond to wideband spectral matrices of wave 1 (resp., wave 2) on components $X$ and $Z$, respectively. We denote them by $\underline{\underline{\Gamma}}_{s, 1_{(X, X)}}$ and $\underline{\underline{\Gamma}}_{s, 1_{(Z, Z)}}$ (resp., $\underline{\underline{\Gamma}}_{s, 2_{(X, X)}}$ and $\underline{\underline{\Gamma}}_{s, 2_{(Z, Z)}}$ ). Blocks (2) and (3) correspond to the cross-spectral matrices of wave 1 (resp., wave 2) containing the interactions between components $X$ and $Z$. We denote them by $\underline{\underline{\Gamma}}_{s, 1_{(X, Z)}}$ and $\underline{\underline{\Gamma}}_{s, 1_{(Z, X)}}$ (resp., $\underline{\underline{\Gamma}}_{s, 2_{(X, Z)}}$ and $\left.\underline{\underline{\Gamma}}_{s, 2(Z, X)}\right)$.

Figure 12 shows two graphs. The upper plot corresponds to the modulus of diagonals of blocks (1) and (4) of $\underline{\underline{\Gamma}}_{s, 1}$. It enables to determine the frequency band $L 1=\left[f_{\text {inf } 1}, f_{\text {sup } 1}\right]$ used to estimate the polarization parameters as well as to carry out the estimation of the amplitude ratio $\widehat{\alpha_{1}}$ between components $X$ and $Z$ (see (25)). The bottom graph of Figure 12 shows the phase of blocks (2) and (3) diagonals. It allows to obtain phase shift $\widehat{\varphi_{1}}$ between components $X$ and $Z$ (see (25)). In a similar way, it is possible to estimate the polarization parameters of wave 2 by using the principal and secondary diagonals of the spectral matrix $\underline{\underline{\Gamma}}_{s, 2}$.

The values obtained for the polarization parameters of each wave are summarized in Table 1 . The phase shift $\varphi$ estimation results are really satisfactory as we find less than $2 \%$ error. The amplitude ratio $\alpha$ is also well estimated with a bigger error $(10 \%)$. This can be attributed to the spectral matrix estimation step. Infact, the spatial smoothing used 


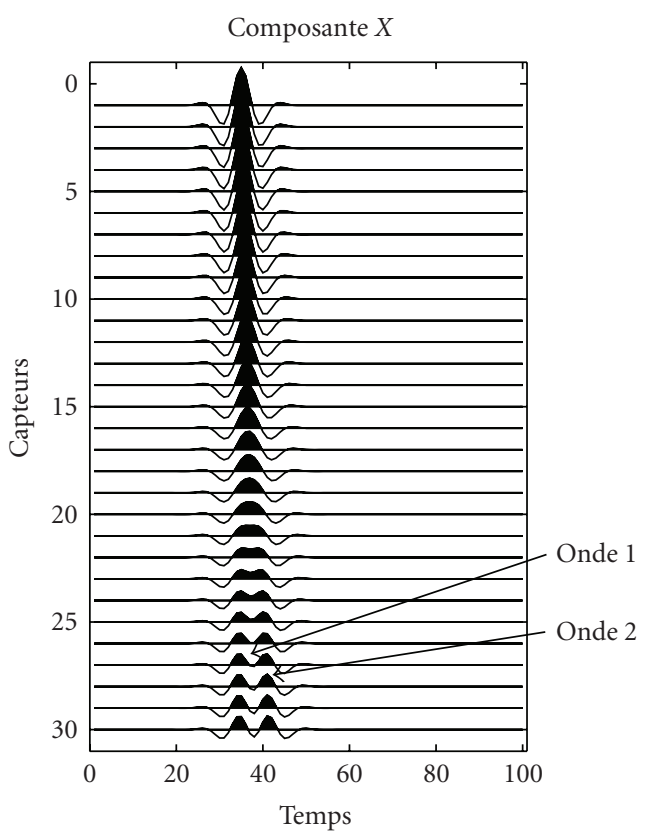

(a)

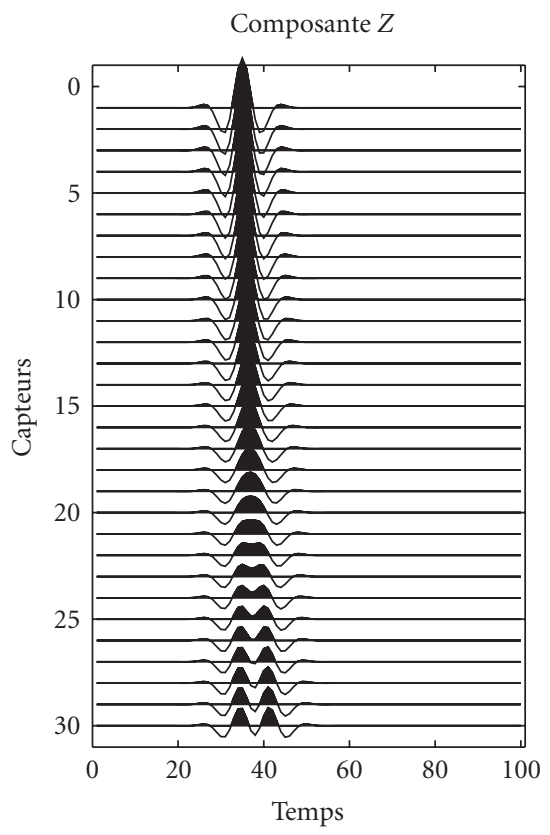

(b)

Figure 15: Model of two waves with close DOAs.

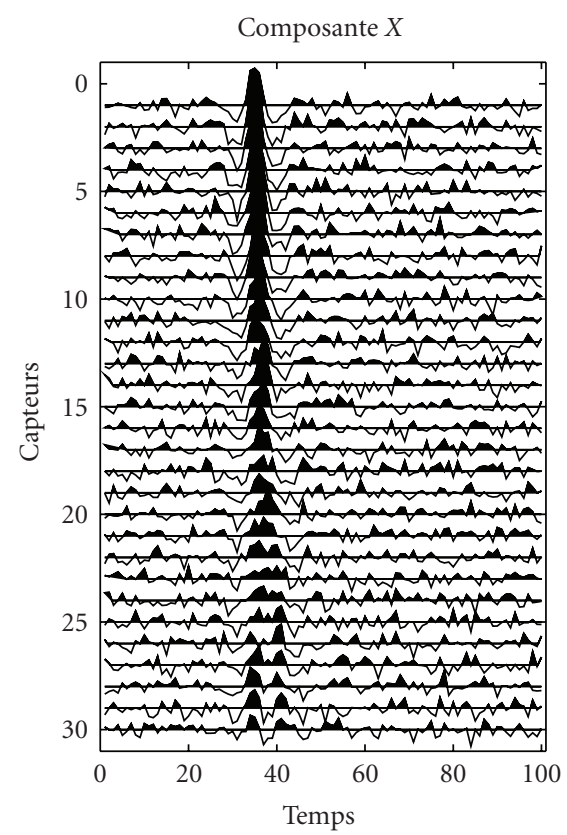

(a)

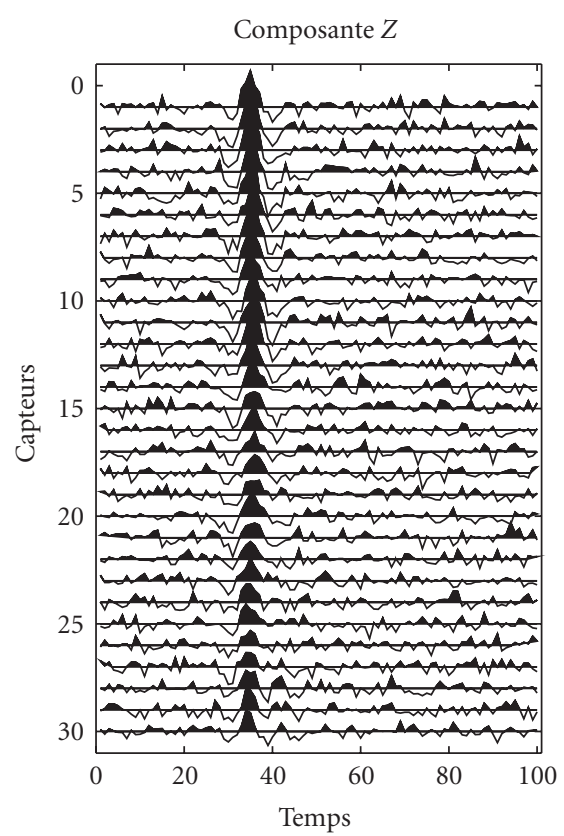

(b)

Figure 16: Initial noisy dataset. to estimate MWSM might affect the wave amplitudes since smoothing is equivalent to an averaging in distance over a small number of sensors.

5.3. DOA Estimation. The last step is the estimation of DOA and offset of each wave (see Section 4). We compute the MW-MUSIC functional for values of the DOA, $\xi(\theta)$, between $[-3 ; 3]$ with a step of 0.01 and for the offset $\tau_{0}$, between $[0 ; 127]$ with a step of 1 . The results obtained are shown in Figure 13. The two main lobes are found at the original values of offsets and DOAs of the two waves samples. Figure 14 corresponds to the cross-sections of the 3D-function (Figure 13) for fixed offsets. The vertical lines show the theoretical values of the DOAs. The estimated values for the DOAs and the offsets are recapitulated in Table 2. One can note that the estimated values are very close to the true values (percentages of error between 0 and $4 \%$ ).

5.4. A More Realistic Example. Now, we consider a 2-Component acquisition simulation recording two waves (15). 


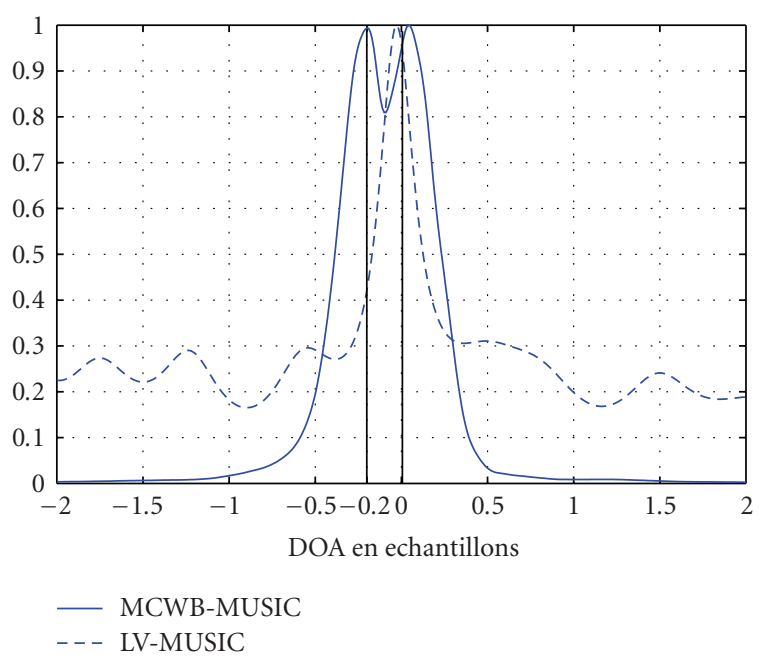

Figure 17: Comparison between MW-MUSIC and LV-MUSIC.

TABLE 2: Estimation of DOAs and offsets.

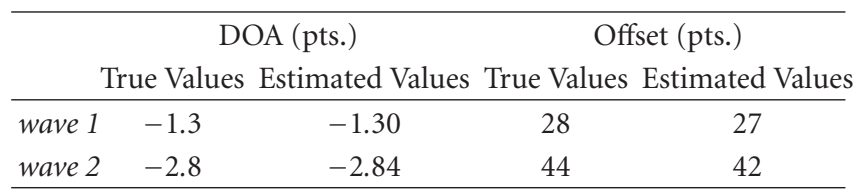

These two waves called Onde1 and Onde2 have shown same spectrum, same offset, same polarisation parameters, and two very close $\mathrm{DOAs}\left(\xi\left(\theta_{\mathrm{O} 1}\right)=0 ; \xi\left(\theta_{\mathrm{O} 2}\right)=-0.2\right)$. The Signal-to-Noise ratio is estimated at $0 \mathrm{~dB}$ (Figure 16).

We propose to compare Long Vector-MUSIC and Multicomponent Wideband-MUSIC algorithms. We show on Figure 17, that resolution given by MW-MUSIC is better than LV-MUSIC. A full separation, polarisation and DOA's estimation have been also realized on a real seismic example [41].

\section{Conclusion}

A novel method providing wavefields separation along with an estimation of both the polarization parameters and the directions of arrival was presented. Taking into account the polarization and the widebandness of the signal leads to a better characterization of a greater number of waves $\left(N_{x} N_{f} N_{c}-1\right)$ as opposed to the monocomponent array case $\left(N_{x}-1\right)$. The performance and efficiency of the method was proven using several simulations. Comparison of the wideband matrix filtering method with those of the classic filtering technique has already been done [15] and gave encouraging result for wideband case. We also obtained promising results for DAO estimation using the proposed method which can be attributed to the fact that our method takes into account the entire frequency information and is therefore insensitive to frequency band selection.

\section{Acknowledgments}

The authors would like to thank reviewers for interesting remarks and A. A. Khan for improving the language.

\section{References}

[1] A. Nehorai and E. Paldi, "Vector-sensor array processing for electromagnetic source localization," IEEE Transactions on Signal Processing, vol. 42, no. 2, pp. 376-398, 1994.

[2] A. Nehorai and E. Paldi, "Acoustic vector-sensor array processing," IEEE Transactions on Signal Processing, vol. 42, no. 9, pp. 2487-2491, 1994.

[3] J. Li and R. T. Compton Jr., "Angle and polarization estimation using ESPRIT with a polarization sensitive array," IEEE Transactions on Antennas and Propagation, vol. 39, no. 9, pp. 1376-1383, 1991.

[4] D. Rahamim, J. Tabrikian, and R. Shavit, "Source localization using vector sensor array in a multipath environment," IEEE Transactions on Signal Processing, vol. 52, no. 11, pp. 30963103, 2004.

[5] H.-W. Chen and J.-W. Zhao, "Wideband MVDR beamforming for acoustic vector sensor linear array," IEE Proceedings: Radar, Sonar and Navigation, vol. 151, no. 3, pp. 158-162, 2004.

[6] K. T. Wong and M. D. Zoltowski, "Self-initiating MUSICbased direction finding and polarization estimation in spatiopolarizational beamspace," IEEE Transactions on Antennas and Propagation, vol. 48, no. 8, pp. 1235-1245, 2000.

[7] K. T. Wong and M. D. Zoltowski, "Diversely polarized rootMUSIC for azimuth-elevation angle-of-arrival estimation," in Proceedings of the AP-S International Symposium \& URSI Radio Science Meeting, pp. 1352-1355, July 1996.

[8] K. T. Wong and M. D. Zoltowski, "Root-MUSIC-based azimuth-elevation angle-of-arrival estimation with uniformly spaced but arbitrarily oriented velocity hydrophones," IEEE Transactions on Signal Processing, vol. 47, no. 12, pp. 32503260, 1999.

[9] K. T. Wong and M. D. Zoltowski, "Uni-vector-sensor ESPRIT for multisource azimuth, elevation, and polarization estimation," IEEE Transactions on Antennas and Propagation, vol. 45, no. 10, pp. 1467-1474, 1997.

[10] M. D. Zoltowski and K. T. Wong, "Closed-form eigenstructure-based direction finding using arbitrary but identical subarrays on a sparse uniform Cartesian array grid," IEEE Transactions on Signal Processing, vol. 48, no. 8, pp. 2205-2210, 2000.

[11] M. D. Zoltowski and K. T. Wong, "ESPRIT-based 2-D direction finding with a sparse uniform array of electromagnetic vector sensors," IEEE Transactions on Signal Processing, vol. 48, no. 8, pp. 2195-2204, 2000.

[12] K. T. Wong and M. D. Zoltowski, "Closed-form direction finding and polarization estimation with arbitrarily spaced electromagnetic vector-sensors at unknown locations," IEEE Transactions on Antennas and Propagation, vol. 48, no. 5, pp. 671-681, 2000.

[13] K. T. Wong, L. Li, and M. D. Zoltowski, "Root-MUSIC-based direction-finding and polarization estimation using diversely polarized possibly collocated antennas," IEEE Antennas and Wireless Propagation Letters, vol. 3, no. 1, pp. 129-132, 2004.

[14] C. Paulus, J. Mars, and P. Gounon, "Wideband spectral matrix filtering for multicomponent sensors array," Signal Processing, vol. 85, no. 9, pp. 1723-1743, 2005.

[15] C. Paulus and J. I. Mars, "New multicomponent filters for geophysical data processing," IEEE Transactions on Geoscience and Remote Sensing, vol. 44, no. 8, pp. 2260-2270, 2006.

[16] H. Mermoz, "Imagerie, corrélation et modèles," Annales des Telecommunications, vol. 31, no. 1-2, pp. 17-36, 1976. 
[17] J. C. Samson, "The spectral matrix, eigenvalues, and principal components in the analysis of multichannel geophysical data," Annales Geophysicae, vol. 1, no. 2, pp. 115-119, 1983.

[18] E. A. Robinson, "Predictive decomposition of time series with application to seismic exploration," Geophysics, vol. 32, pp. 418-484, 1967.

[19] B. D. Rao and K. V. S. Hari, "Weighted subspace methods and spatial smoothing. Analysis and comparison," IEEE Transactions on Signal Processing, vol. 41, no. 2, pp. 788-803, 1993.

[20] S. S. Reddi, "On a spatial smoothing technique for multiple source location," IEEE Transactions on Acoustics, Speech, and Signal Processing, vol. 35, no. 5, p. 709, 1987.

[21] T. Shan, M. Wax, and T. Kailath, "On spatial smoothing for direction-of-arrival estimation of coherent signals," IEEE Transactions on Acoustics, Speech, and Signal Processing, vol. 33, no. 4, pp. 806-811, 1985.

[22] S. U. Pillai and B. H. Kwon, "Forward/backward spatial smoothing techniques for coherent signal identification," IEEE Transactions on Acoustics, Speech, and Signal Processing, vol. 37, no. 1, pp. 8-15, 1989.

[23] H. Wang and M. Kaveh, "Coherent signal-subspace processing for the detection and estimation of angles of arrival of multiple wide-band sources," IEEE Transactions on Acoustics, Speech, and Signal Processing, vol. 33, no. 4, pp. 823-831, 1985.

[24] N. Thirion, J. I. Mars, and J.-L. Lacoume, "Analytical links between steering vectors and eigenvectors," in Proceedings of the European Signal Processing Conference (EUSIPCO '96), pp. 81-84, Trieste, Italy, 1996.

[25] N. Thirion, J. Lacoume, and J. Mars, "Resolving power of spectral matrix filtering: a discussion on the links steering vectors / eigenvectors," in Proceedings of the 8th IEEE Signal Processing Workshop on Statistical Signal and Array Processing (SSAP'96), pp. 340-343, June 1996.

[26] N. Thirion, J. I. Mars, and J.-L. Boelle, "Separation of seismic signals: a new concept based on blind algorithm," in Proceedings of the European Signal Processing Conference (EUSIPCO '96), pp. 85-88, Trieste, Italy, 1996.

[27] R. N. Jolly, "Investigation of shear waves," Geophysics, vol. 21, no. 4, pp. 905-938, 1956.

[28] M. Shimshoni and S. W. Smith, "Linear end parabolic $\tau$-p revisited," Geophysics, vol. 29, no. 5, pp. 664-671, 1964.

[29] S. A. Greenhalgh, I. M. Mason, C. C. Mosher, and E. Lucas, "Seismic wavefield separation by multicomponent taup polarisation filtering," Tectonophysics, vol. 173, no. 1-4, pp. 53-61, 1990.

[30] C.-S. Shieh and R. B. Herrmann, "Ground roll: rejection using polarization filters," Geophysics, vol. 55, no. 9, pp. 1216-1222, 1990.

[31] G. M. Jackson, I. M. Mason, and S. A. Greenhalgh, "Principal component transforms of triaxial recordings by singular value decomposition," Geophysics, vol. 56, no. 4, pp. 528-533, 1991.

[32] E. A. Flinn, "Signal analysis using rectilinearity and direction of particule motion," Proceedings of the IEEE, vol. 56, pp. 18741876, 1965.

[33] C. B. Archambeau and E. A. Flinn, "Automated analysis of seismic radiation for source characteristics," Proceedings of the IEEE, vol. 53, pp. 1876-1884, 1965.

[34] R. O. Schmidt, A signal subspace approach to multiple emitter location and spectral estimation, Ph.D. dissertation, Stanford University, Stanford, Calif, USA, 1981.
[35] R. O. Schmidt, "Multiple emitter location and signal parameter estimation," IEEE Transactions on Antennas and Propagation, vol. 34, no. 3, pp. 276-280, 1986.

[36] M. Kaveh and A. J. Barabell, "The statistical performance of the MUSIC and the minimum-norm algorithms in resolving plane waves in noise," IEEE Transactions on Acoustics, Speech, and Signal Processing, vol. 34, no. 2, pp. 331-341, 1986.

[37] P. Stoica and A. Nehorai, "MUSIC, maximum likelihood, and Cramer-Rao bound," IEEE Transactions on Acoustics, Speech, and Signal Processing, vol. 37, no. 5, pp. 720-741, 1989.

[38] E. R. Ferrara Jr. and T. M. Parks, "Direction finding with an array of antennas having diverse polarizations," IEEE Transactions on Antennas and Propagation, vol. 31, no. 2, pp. 231-236, 1983.

[39] Y. Hua, "Pencil-MUSIC algorithm for finding twodimensional angles and polarizations using crossed dipoles," IEEE Transactions on Antennas and Propagation, vol. 41, no. 3, pp. 370-376, 1993.

[40] A. Swindlehurst and M. Viberg, "Subspace fitting with diversely polarized antenna arrays," IEEE Transactions on Antennas and Propagation, vol. 41, no. 12, pp. 1687-1694, 1993.

[41] C. Paulus and J. I. Mars, "Polarization analysis on seismic data after multicomponent wavefield filtering," in Proceedings of EAGE/SEG Research Workshop, Multicomponent SeismicPast, Present and Future, Pau, France, September 2005. 